\title{
P-3
}

\section{Application of Multivariate Process Control (MPC) in the Natural Rubber Industry}

\author{
Gunaratne M.D.N. ${ }^{1 *}$, Wijesuriya W. ${ }^{2}$ and Amarasekera D.A.B.N. ${ }^{3}$ \\ ${ }^{1}$ University of Moratuwa, Katubedda, Sri Lanka \\ ${ }^{2}$ Rubber Research Institute, Agalawatta, Sri Lanka \\ ${ }^{3}$ University of Ruhuna, Sri Lanka \\ *mdng2012@gmail.com
}

\begin{abstract}
Statistical process control (SPC) monitors specified quality characteristics of a product or service to detect whether the process has changed in a way that would affect product quality and to measure the current quality of products or services. This study introduces several applications on multivariate process control in the rubber industry employing the factory data in the Dartonfield estate of the Rubber Research Institute of Sri Lanka (RRI). Multivariate process control was applied to consider two or more variables simultaneously. In this study multivariate techniques were done for the application of bleaching agents, sodium bisulphate and acids in the process of crepe rubber production.

Multivariable scatter diagrams and control ellipse has been used to show the process when the multivariate data composed of two variables. Hotelling T2 control chart was used to monitor application of chemicals simultaneously in a single chart. Principal components were used to interpret dimensions of components and star charts were applied to diagnostic for determining the relative contributions of the each chemical component. The multivariate EWMA (MEWMA) control chart for monitoring the process mean vector was a straightforward extension of the univariate EWMA chart. The graphical presentation is very important and it is a quick way to see the advantages of MPC.

Multivariate Statistical analysis is concerned with data that consist of sets of measurements on a number of variables. So, multivariate quality control provides a way for monitoring and evaluation of chemical application process. Scatter plots and control ellipse is very important to detect out of control points and relationships as a graphical method. Hotelling T2 is an important chart that can be decomposed into an overall measure of distance of the group means from the target T2 and measure of variability. Principal components can be useful for multivariate quality control, especially to observe variation of dimensions of chemical applications. Multivariate EWMA procedure use additional information from recent history of the process and it is more sensitive in monitoring chemical application process.
\end{abstract}

Therefore multivariate process control provides way for manufacturer to test their products in an environment that provide many advantages over univariate models.

Keywords: Rubber, Univariate, Multivariate, Process control, Statistical quality control

Proceedings of the International Forestry and Environment Symposium 2013 of the Department of Forestry and Environmental Science, University of Sri Jayewardenepura, Sri Lanka. 